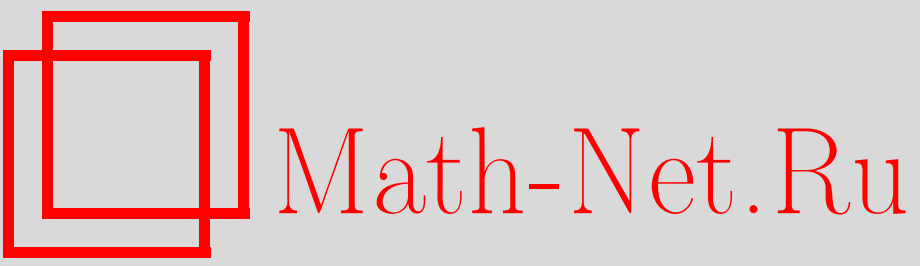

Г. А. Айгунов, О максимально возможной скорости роста решений задачи Коши и нормированных собственных функций одного класса нелинейных операторов типа Штурма-Лиувилля с непрерывной положительной весовой функцией, УМH, 2000, том 55, выпуск 2, 129-130

DOI: https://doi.org/10.4213/rm272

Использование Общероссийского математического портала Math-Net.Ru подразумевает, что вы прочитали и согласны с пользовательским соглашением

http://www.mathnet.ru/rus/agreement

Параметры загрузки:

IP : 54.237 .59 .107

26 апреля 2023 г., 16:44:12 


\title{
О МАКСИМАЛЬНО ВОЗМОЖНОЙ СКОРОСТИ РОСТА РЕШЕНИЙ ЗАДАЧИ КОШИ И НОРМИРОВАННЫХ СОБСТВЕННЫХ ФУНКЦИЙ ОДНОГО КЛАССА НЕЛИНЕЙНЫХ ОПЕРАТОРОВ ТИПА ШТУРМА-ЛИУВИЛЛЯ С НЕПРЕРЫВНОЙ ПОЛОЖИТЕЛЬНОЙ ВЕСОВОЙ ФУНКЦИЕЙ
}

\author{
Г. А. Айгунов
}

Пусть $0<m<M<\infty$ - фиксированные числа. Обозначим через $C_{[0,1]}^{+}$множество всех непрерывных функций $\rho(x)$ на сегменте $[0,1]$, удовлетворяющих условию $m \leqslant \rho(x) \leqslant M$. На множестве $C_{[0,1]}^{+}$рассмотрим обычную $C$-метрику. В дальнейшем такие функции будем называть весовыми функциями.

Пусть $p>1$, тогда по определению будем полагать $(z)_{p} \equiv|z|^{p-1} \operatorname{sign} z=|z|^{p-2} z$. Рассмотрим задачу Коши

$$
\begin{gathered}
-\frac{d}{d x}\left(y^{\prime}(x)\right)_{p}=\lambda \rho(x)(y(x))_{p}, \quad 0 \leqslant x \leqslant 1, \quad \lambda>0, \\
y\left(x_{0}\right)=a_{0}, \quad y^{\prime}\left(x_{0}\right)=b_{0}, \quad a_{0}^{2}+b_{0}^{2}=1, \quad x_{0} \in[0,1] .
\end{gathered}
$$

Решение задачи (1) существует, единственно и непрерывно зависит от начальных данных, параметра $\lambda$ и весовой функции $\rho(x)$, см. [1].

Представляется целесообразным рассмотреть спектральную задачу, которая возникает в ряде задач, связанных, прежде всего, с проблемами колебаний неоднородной среды, а также с вопросами приближения гладких классов функций:

$$
\begin{gathered}
-\frac{d}{d x}\left(y^{\prime}(x)\right)_{p}=\lambda \rho(x)(y(x))_{p} \quad(0<x<1), \\
y(0)=y(1)=0, \quad \int_{0}^{1} \rho(x) y^{2}(x) d x=1 .
\end{gathered}
$$

Л.А. Люстерник [2] показал, что штурмовская теория собственных значений может быть в большей мере перенесена на спектральню задачу (2). А. Элбертом [1] было выяснено, что спектр задачи (2) дискретен, состоит из простых собственных чисел и асимптотически $\lambda_{k} \sim C_{p} k^{p}$ при $k \rightarrow \infty\left(C_{p}>0\right)$.

Обозначим через $y_{k}(x)$ собственную функцию, соответствующую собственному числу $\lambda_{k}$ $(k=1,2, \ldots)$. Условимся говорить, что совокупность собственных функций $y_{k}(x)(k=1,2, \ldots)$ ассоциирована с весовой функцией $\rho(x)$ спектральной задачи $(2)$.

В данной работе исследовано асимптотическое поведение не только нормированных собственных функций нелинейной краевой задачи типа Штурма-Лиувилля, но и решений задачи Коши в зависимости от параметра $\lambda$. Причем исследование асимптотического поведения собственных функций нелинейной краевой задачи типа Штурма-Лиувилля проводится, исходя из асимптотического поведения решения задачи Коши. Доказанные теоремы дают неулучшаемые оценки для норм собственных функций в классе весовых функций $\rho(x) \in C_{[0,1]}^{+}$.

Пусть $\alpha(\lambda)>0$ непрерывная на $(0, \infty)$ функция, стремящаяся к нулю при $\lambda \rightarrow \infty$, а $\Phi_{\lambda}(\rho)$ функционал, определенный соотношением

$$
\Phi_{\lambda}(\rho) \equiv \frac{\|y(x, \lambda, \rho)\|_{C_{[0,1]}}}{\left(\int_{0}^{1} \rho(x) y^{2}(x, \lambda, \rho) d x\right)^{1 / 2}} .
$$

Теорема 1. 1) Для любой весовой функции $\rho(x) \in C_{[0,1]}^{+}$справедливо равенство

$$
\lim _{\lambda \rightarrow \infty} \lambda^{-1 /(2 p)} \Phi_{\lambda}(\rho)=0 .
$$

2) В любом шаре из $C_{[0,1]}^{+}$существует весовая функция $\rho_{0}(x)$ такая, что

$$
\lim _{\lambda \rightarrow \infty} \alpha^{-1}(\lambda) \lambda^{-1 /(2 p)} \Phi_{\lambda}\left(\rho_{0}\right)=\infty .
$$


Ввиду того, что $\alpha(\lambda)$ может быть выбрана произвольно, теорема 1 устанавливает точные оценки (асимптотические) для $\Phi_{\lambda}(\rho)$ в классе весовых функций из $C_{[0,1]}^{+}$.

ТЕОРема 2. Пусть функция $\rho(x) \in C_{[0,1]}^{+}$является функцией ограниченной вариации. Тогда функционал $\Phi_{\lambda}(\rho)$ равномерно по всем $\lambda>0$ ограничен.

Рассмотрим спектральную задачу (2). Справедлива

Теорема 3. Имеют место следующие утверждения.

1) Пусть $\rho(x) \in C_{[0,1]}^{+}$, mогдa $\lim _{n \rightarrow \infty} \lambda_{n}^{-1 /(2 p)}\left\|y_{n}(x, \rho)\right\|_{C_{[0,1]}}=0$.

2) Пусть $\rho(x) \in C_{[0,1]}^{+}$, тогда в любой $C$-окрестности $\rho(x)$ существует весовая функиия $\rho_{0}(x, \alpha) \in C_{[0,1]}^{+} \cap C_{[0,1)}^{\infty}$ такая, что

$$
\varlimsup_{n \rightarrow \infty} \alpha^{-1}\left(\lambda_{n}\right) \lambda_{n}^{-1 /(2 p)}\left\|y_{n}(x, \rho)\right\|_{C_{[0,1]}}=\infty .
$$

Из теоремы 3 следует, что в классе бесконечно дифференцируемых на $[0,1)$ весовых функций максимально возможная скорость роста собственных функций по спектральному параметру $\lambda$ такая же, как и во всем объемлющем классе $C_{[0,1]}^{+}$. Поэтому возникает вопрос о зависимости асимптотического поведения собственных функций от гладкости весовой функции $\rho(x)$ в некоторой точке, в частности, в точке $x=1$.

Заметим, что вместо точки $x=1$ можно взять любую точку $x_{0} \in[0,1]$, точка $x=1$ рассматривается лишь для определенности выкладок при доказательстве теоремы. Справедлива следующая

ТеОрема 4. Пусть $\rho(x) \in C_{[0,1]}^{+}, r-$ вещественное число. Тогда имеют место следующие утвержсдения.

1) В любой $C$-окрестности весовой функиии $\rho(x)$ существует весовая функция $\rho_{0}(x) \equiv \rho_{0}(x, \alpha, r) \in C_{[0,1]}^{+} \cap C_{[0,1)}^{\infty}$ mакал, что $\rho_{0}^{\prime}(1)=r u$

$$
\varlimsup_{n \rightarrow \infty} \alpha^{-1}\left(\lambda_{n}\right) \lambda_{n}^{-1 /(2 p)}\left\|y_{n}\left(x, \rho_{0}\right)\right\|_{C_{[0,1]}}=\infty .
$$

2) В любой C-окрестности весовой функции $\rho(x)$ существует весовая функция $\rho_{0}(x, \alpha) \in C_{[0,1]}^{+} \cap C_{[0,1)}^{\infty}$ такая, что $\rho_{0}(x)$ не дифференцируема в точке $x=1$ и

$$
\varlimsup_{n \rightarrow \infty} \alpha^{-1}\left(\lambda_{n}\right) \lambda_{n}^{-1 /(2 p)}\left\|y_{n}\left(x, \rho_{0}\right)\right\|_{C_{[0,1]}}=\infty
$$

СледСтвиЕ. Порядок роста нормированных собственных функций спектральной задачи (2) в классе весовых функций, имеющих первую производную, в метрике $C$ такой же, как и во всем обвемлющем классе $C_{[0,1]}^{+}$.

Таким образом, нам удалось выявить наиболее узкий класс весовых функций, для которых возможен максимально быстрый рост нормированных собственных функций.

Более того, из теорем 3,4 следует, что нарушение непрерывности первой производной в одной точке влечет за собой нарушение равномерных оценок нормированных собственных функций и при этом достигается максимально возможная скорость их роста, как и в классе $C_{[0,1]}^{+}$.

В заключение заметим, что аналогичные теоремы рассмотрены в работах [3], [4].

\section{СПИСОК ЛИТЕРАТУРЫ}

[1] Elbert A. // Colloq. Math. Soc. János Bolyai. 1981. V. 30. P. 153-180. [2] Люстерник Л. А. // Докл. АН СССР. 1937. Т. 15. С. 235-238. [3] Гехтман М. М., Загиров Ю. М. // УМН. 1992. Т. 47. № 3. С. 157-158. [4] Айгунов Г. А. // УМН. 1997. Т. 52. №6. С. 147-148. 